\title{
Effect of Aerosil and Oleic Acid on Sedimentation of Magnetorheological Fluid
}

\author{
HirenPrajapati, Jas Shahanand ,HitarthNimkar
}

\begin{abstract}
Magnetorheological Fluids (MRFs) are considered as smart fluids because they control viscosity using external magnetic field. It contains ferro-magnetic powder which are aligned in magnetic flux lines. The magnetic force between particles are controlled by magnetic field intensity. This controllable viscosity makes them acceptable in many mechanical applications, but due to difference in density between suspended particles and carrier fluid sedimentation is bound to occur. This thus creates the need of some additives. In our study, silica Nano particles (commercially known as Aerosil 200) is used as stabilizer and Oleic Acid is used as surfactant and their effect on sedimentation is studied in this article. Some other synthesis parameters like particle concentration, stirring duration and material loading also cause some change in sedimentation rate.
\end{abstract}

Keywords: Magnetorheological Fluid, settling, Synthesis, sedimentation.

\section{INTRODUCTION}

Magnetorheological fluids (MRFs) are a class of smart materials. MRF consists micro sized iron particles dispersed in viscous carrier fluid.[1] These dispersed iron particles makes chain like structures in presence of magnetic field and converts into semisolids from liquid state. The formed particle-chains restrict fluid movement and increase yield strength of MR fluid. After removing magnetic field the semisolid form converts into fluid state back within milliseconds again. [2] Total viscosity (or Dynamic Yield Stress) of MRF is the viscosity in the presence of magnetic field and also the viscosity of MRF in absence of the magnetic field. Therefore MRFs are considered as nonNewtonian fluids. This rapid change of MRF viscosity in presence of magnetic field makes them suitable in lot many applications like isolators, shock absorbers, clutches, engine mounts, alternators, power steering pumps, control valves, brakes, dampers etc.[3] The operating range of said applications can be changed using MRF. The stability of iron particles, within carrier fluid is one of the problems of largest interest from the point of view of the technological applications of these systems. It can interfere with the magnetorheological response because of the non-uniform distribution of particles. Small particle size and high viscosity carrier fluids are recommended to reduce sedimentation but requirement of low viscosity in absence of magnetic field makes the problem more difficult as MRF

HirenPrajapati, Asst. Professor, Mechanical Engineering Department, Institute of Technology, Nirma University, Ahmedabad, India.Email: hp.cspit@gmail.com

Jas Shahanand, Mechanical Engineering Department, Institute of Technology, Nirma University, Ahmedabad, India Email:jasshahanand@gmail.com

HitarthNimkar, Mechanical Engineering Department, Institute of Technology, Nirma University, Ahmedabad, India.. Email: hitarthnimkar@gmail.com
Revised Manuscript Received on 14 September, 2019.

viscosity and yield strength are dependent on particle materials, particle concentration, particle size and size distribution, applied magnatic field, carrier fluids etc. [2-8]

Different approaches are attempted to control sedimentation:

(a) adding thixotropic agents (carbon fibers, grease [9], silica nanoparticles[10])

(b) adding surfactants (oleic [5], stearic acid)

(c) adding magnetic nanoparticles [11]

(d) the use of viscoplastic media as continuous phase [2]

(e) water in oil emulsions as continuous phase. [8][10]

The most popular stabilizers are grease [9] and fumed silica [1][10]

One another category of magnetic fluid is ferrouid (FF) which consists dispersion of Nano sized iron oxide particles. This reduces sedimentation problems because there is less density difference between particles and carrier uid. The basic difference between FF (10nm) and MRF (in micron) is the size of the particles. [8][11] The particles are suspended by Brownian motion in FF. In usual magnetic field brownian forces dominates the magnetic forces. [15] Therefore FF offers very less change in viscosity under very high magnetic fields compared to MRF. This make their application limited where small viscosity change is required.[8] That is why FF used in magnetic printers. In contrast, high magnetoviscouse response of MRF make them usable in wide technical application. [12]

The proposed work will focus on synthesis of MRF using Electrolytic Iron Powder, carrier fluids, and additives to reduce sedimentation rate.

\section{MATERIALS AND SYNTHESIS OF MAGNETORHEOLOGICAL FLUID}

In this work Electrolytic Iron Powder (EIP), Silicon oil, Aerosil and Oleic acid are used as suspension, carrier fluid, stabilizer and surfactant. Electrolytic Iron powder is provided by Industrial Metal Powder, Pune and used without any further processing. EIP is characterized by shape and size distribution on Field Emission Scanning Electron Microscope (FESEM) and on Helos Particle Size Analysis Windox 5 respectively. Fig 1 shows SEM image of EIP which indicates needle shape of particles. The particle shape plays important role in yield stress of MRF. The needle shaped particles offer more surface area. Therefore offers more yield stress when they rub against rotating disk in rheometer.[13] Mean size of 


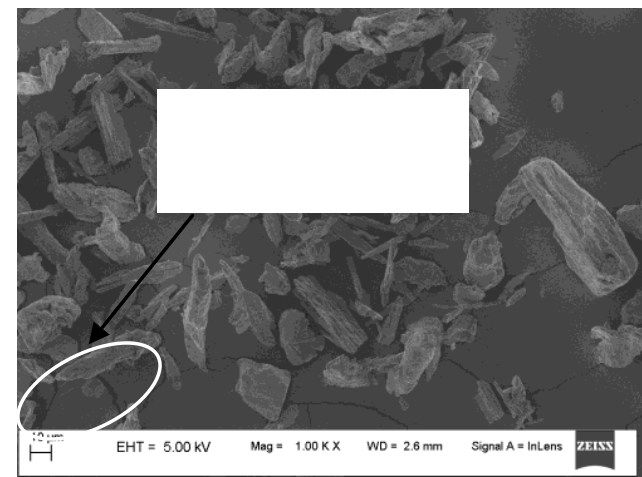

Fig. 1. Scanning electron microscope picture of Electrolytic Iron Powder with bar size 10 micron

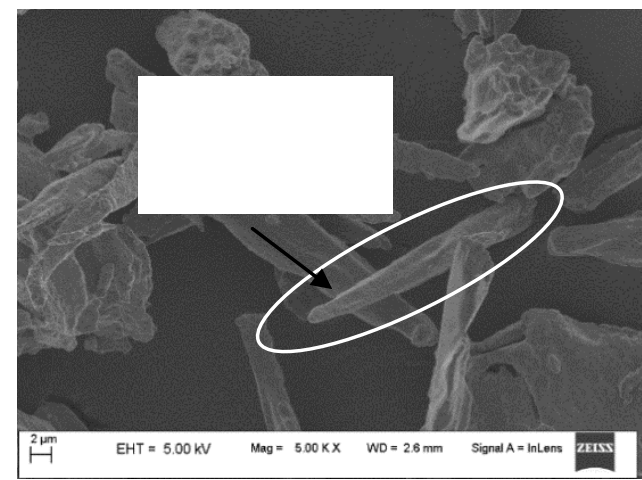

Fig. 2. Scanning electron microscope picture of Electrolytic Iron Powder with bar size 10 micron

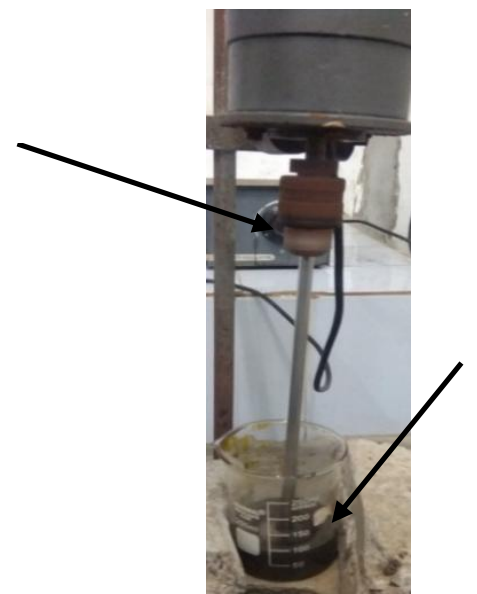

Fig. 3.Magnetorheological Fluid (MRF) synthesis.

Electrolytic Iron Powder is 20 micron from Helos Particle Size Analyzer. The silicon oil is used as carrier fluid having viscosity $300 \mathrm{cst}$ and density $0.97 \mathrm{gm} / \mathrm{ml}$. Aerosil 200 has density $0.084 \mathrm{gm} / \mathrm{cm} 3$. Oleic acid is used as surfactant which may also play some role in sedimentation as well. It has a density of $0.889 \mathrm{gm} / \mathrm{ml}$.

Synthesis of Magnetorheological Fluid (MRF) is performed using mechanical stirrer. Synthesis involves different variables which can play a major role in sedimentation of MRF. These variables (ingredient addition, stirring speed, stirring time etc.) are observed in sedimentation study of MRF in this article. MRF samples are synthesised by two different methods and their effect on sedimentation is also studied. In first method silicon oil, particles, oleic acid and aerosol are kept in glass beaker and stirred using mechanical stirrer. In second method all the members of MRF are mixed stage by stage. The oleic acid, aerosil and particles are mixed in silicon in said order. The stirring set up is shown in fig. 3 .

\section{EXPERIMENTATION\& METHODOLOGY}

The major constituent of magnetorheological fluid (MRF) is carrier fluid. It works as medium for suspending magnetic particles and other additives. In present work silicon oil is used as carrier fluid which is colourless and oleic acid is also colourless. Therefore addition of this two constituent formulates colourless solution. Aerosil is white in colour hence addition of Aerosil produces white turbid solution Later when Electrolytic Iron powder is added, it changes to dark grey colour. It is observed when MRF is allowed to settle then particle separation cause clear boundary separation between clear (absence of particles) and turbid part (containing particles) as shown in fig 4(a). Now movement of boundary separation is measured with time which gives sedimentation speed. In our work, borosil graduated cylinder is used for filling the MRF sample which has an internal diameter of $30 \mathrm{~mm}$ and capacity of $50 \mathrm{ml}$. The sedimentation rate is measured by taking ratio of clear part (a) and total sample height.[14] Sedimentation ratio (SR) can be given as:

$$
S R=\frac{a}{(a+b)}
$$

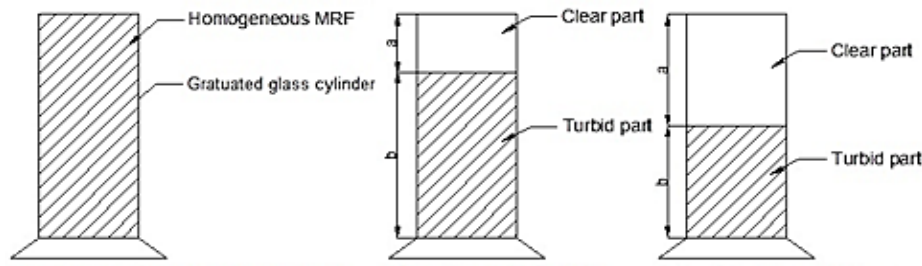

(a) Progressive sedimentation

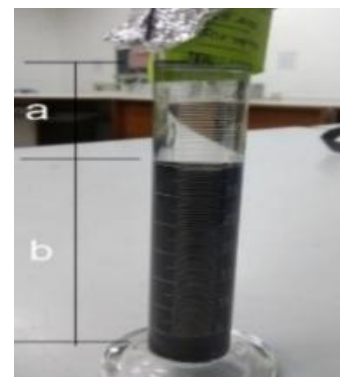

(b) Settled sample

Fig. 4 Progressive sedimentation

\section{RESULTS AND DISCUSSION}

This chapter contains effect of Aerosil, Oleic acid and some other synthesising parameters on stability of silicon oil based magnetorheological fluid.

\section{A. Effect of particle concentration}

General hypothesis when particle concentration is increased, it also increases particle density in sample, which 
should also increase particle settling rate. However sedimentation rate reduces with increase in particle concentration as shown in fig 5 . This may be due to increase in particle concentration as it causes large particles to settle rapidly but as particle size in not uniform as small particles are also present in the sample. Therefore settling of large velocity. This fluid flow causes suspension of lighter density particles.[15]

\section{B. Effect of Aerosil 200}

Three different samples are prepared to study the effect of Aerosil as stabilizer. All the samples contain $20 \%$ EI and stirred for 2 hours at $1500 \mathrm{rpm}$. It is clear from the fig 6 percentage addition of Aerosil improves the stability of MRF.

\section{C. $\quad$ Effect of oleic acid}

Oleic acid is used as a surfactant. It is generally used to reduce particle agglomeration. However it can also change sedimentation behaviour of MRF. Therefore three sample are prepared with different concentration of Oleic Acid in absence of Aerosil with 2 hour stirring at $1500 \mathrm{rpm}$. Here our intuition turned to correct and oleic acid causes variation in stability of MRF as shown in fig. 7. This behaviour due to oleic acid might not be able covered surface of magnetic particles in initial 4 hours. Once particles are coated completely then agglomeration stops and stable MRF is achieved.[16]

D. Combined effect of Oleic acid and Aerosil based $M R F$

Only stabilizers are not sufficient to reduce abrasive wear, agglomeration and corrosion of particles surfactant are also to be used. Therefore to study combined effect, sample with both surfactant and stabilizer is prepared and its sedimentation observed. It is clear from fig. 8 due to presence of oleic acid with Aerosil stability of MRF reduces compared to MRF without Oleic Acid. But here we have to compromise in stability for long life of MRF.

\section{E. Effect of stirring duration}

Stirring duration is important for homogeneous mixing of all the constituent of MRF. In literatures stirring hours are used above 12 hours but such a long duration reduces productivity of MRF. Therefore here we have prepared MRF with three different stirring duration. When compared to a 10 hours stirred sample against a 2 hours stirred sample, the initial result as well as the result after 6 hours seems to be similar, with slight variation in the middle. Hence in all our study, the sample were stirred for 2 hours only. The results of these are plotted in fig 9.

F. Comparison of instantaneous and gradual addition of MRF constituent

Two different approaches are used in synthesis of MRF. In first approach all the MRF constituents are added and stirred for two hours. In second approach in first half an hour oleic acid is mixed with silicon only, then in the next half an hour previously prepared solution is stirred with Aerosil and finally EI powder is stirred for one hour. Both these samples are observed and more stability is found for particles causes upward flow of carrier fluid with high

MRF in which constituents are added gradually.

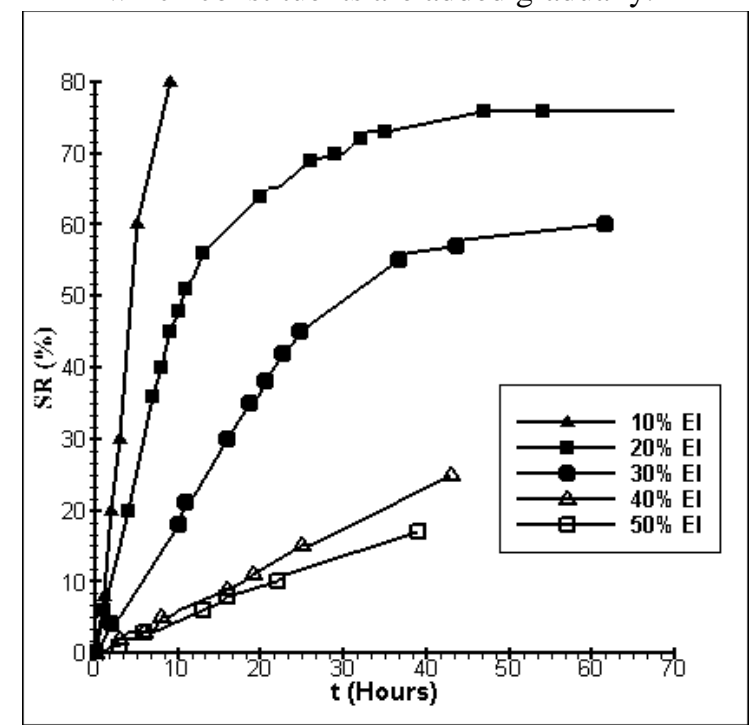

Fig. 5. Effect of particle concentration on sedimentation

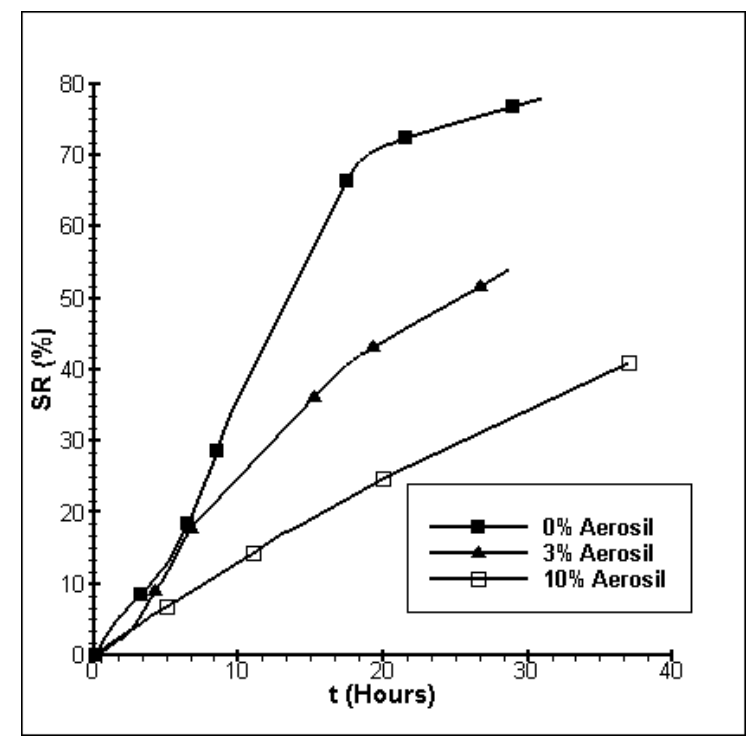

Fig. 6. Effect of Aerosil on sedimentation

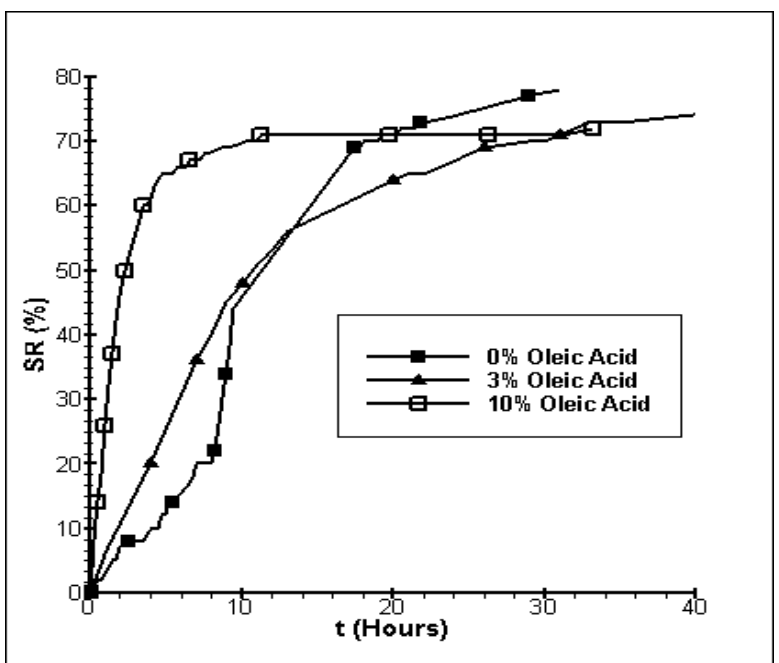

Fig. 7. Effect of Oleic Acid on sedimentation 


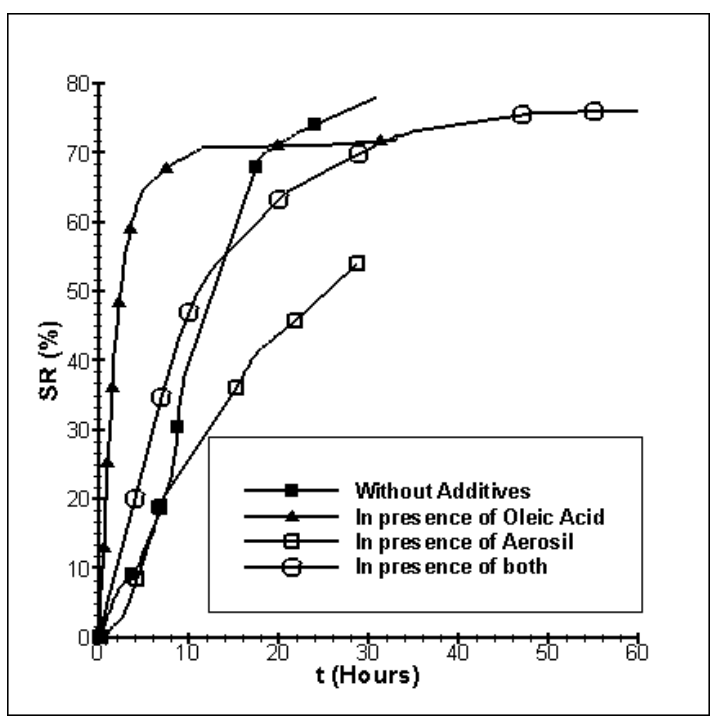

Fig. 8. Combined effect of Aerosil and Oleic Acid on sedimentation

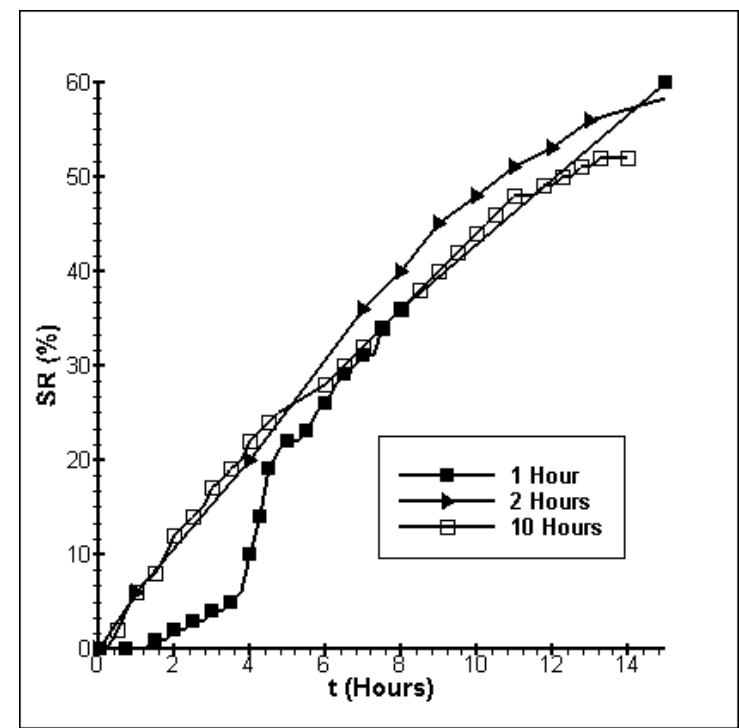

Fig. 9. Effect of stirring hours on sedimentation

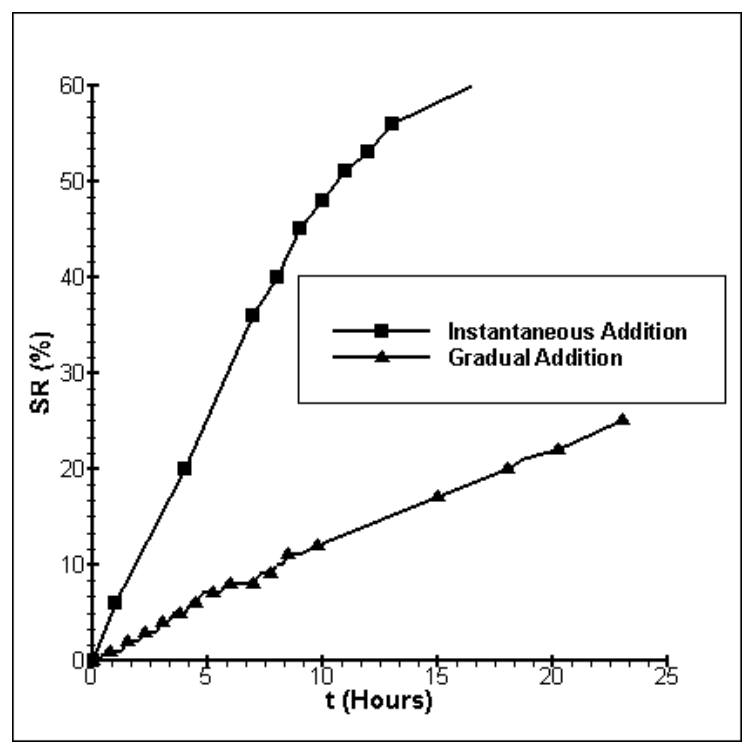

Fig. 10. Effect of synthesising method on sedimentation

\section{CONCLUSION}

Additives are an important part of Magnetorheological Fluids (MRF). There is improvement in stability of MRF in presence of Aerosil but addition of surfactant (here oleic acid) causes reduction in stability but for long life of MRF, surfactants are an important constituent. Therefore some compromise has to be made in stability. During synthesis gradual addition of all the constituent gives remarkable improvement in stability. The stirring duration is also playing major role in the stability. Higher the stirring duration, higher is the stability. However this philosophy works well up to certain extent. There is no significant improvement in the stability after homogeneous suspension and complete particle coating of surfactant. Therefore 10 hours and 2 hours stirring are having marginal impact on the results. Sedimentation study based on visual observation is a qualitative way. Such method cannot give quantitative results. It is observed that some iron particles stick to the wall of measuring cylinder and that hinders the actual sedimentation at the interior portion. Therefore more accurate and reliable method has to be found out for quantitative study.

\section{ACKNOWLEDGMENT}

Financial support by Nirma University Funded Minor Research Project (NU/Ph.D/MRP/IT/18-19/538) is gratefully acknowledged.

\section{REFERENCES}

1. Ashtiani M., Hashemabadi SH. and Ghaffari A.: A review on the magnetorheo-logical fluid preparation and stabilization. Journal of magnetism and Magnetic Materials, 374, pp.716-730 (2015).

2. Carlson J D.: What makes a good MR fluid? Journal of intelligent material systems and structures, 13(7-8): pp.431-435 (2002).

3. Gadekar P., Kanthaleand VS. andKhaire ND.. Magnetorheological fluid and its applications. International Journal of Current Engineering and Technology (2017).

4. Jolly M. R., Bender J. W., and Carlson J. D.: Properties and applications of commercial magnetorheological fluids. Journal of intelligent material systems and structures, 10(1): pp.5-13 (1999)

5. Lim S. T., Cho M. S., Jang I. B., and Choi H. J.: Magnetorheological characterization of carbonyl iron based suspension stabilized by fumed silica. Journal of magnetism and magnetic materials, 282: pp.170-173 (2004).

6. Saraswathamma K., Jha S., and Rao P. V.: Rheological characterization of MR polishing fluid used for silicon polishing in BEMRF process. Materials and Manufacturing Processes, 30(5): pp.661-668 (2015).

7. Sidpara A., Das M., and Jain VK..: Rheological characterization of magnetorheological finishing fluid. Materials and Manufacturing Processes, 24(12): pp.14671478 (2009).

8. Olabi AG. andGrunwald A.: Design and application of magnetorheological fluid. Materials \& design, 28(10): pp. 2658-2664 (2007) 
9. Shetty BG. and Prasad P.S.S.: Rheological properties of a honge oil based magnetorheological fluid used as carrier liquid. Defence Science Journal, 61(6): pp. 583-589 (2011).

10. Lopez-Lopez MT, Vicente J. D., Caballero F. G., and Duran J..: Stability of magnetizable colloidal suspensions by addition of oleic acid and silica nanoparticles. Colloids and Surfaces A: Physicochemical and Engineering Aspects, 264(1-3): pp. 75-81 (2005).

11. Yang Y., Li L., and Chen G.: Static yield stress of ferrofluid based magnetorheological fluids. Rheologicaacta, 48(4): pp. 457-466 (2009).

12. Lopez-Lopez MT., Kuzhir P., Lacis S., Bossis G., Gonzalez-Caballero F., and Duran J.: Magnetorheology for suspensions of solid particles dispersed in ferrouids. Journal of Physics: Condensed Matter, 18(38):S2803 (2006).

13. Sarkar C. \&Hirani. H.: Effect of Particle Size on Shear Stress of Magnetorheological Fluids, Smart Science, 3:2, pp. 65-73, DOI: 10.1080/23080477. 11665638 (2015).

14. Turczyn R and Kciuk M.: Preparation and study of model magnetorheological fluids. Journal of Achievements in Materials and Manufacturing Engineering, 27(2): pp. 131134 (2008)

15. Coulson J, Richardson F.: Chemical Engineering Particle technology \& separation processes, 5 edition, volume 2. Elsevier (2003).

16. Bossis, $\mathrm{G}$ and Volkova, $\mathrm{O}$ and Lacis, $\mathrm{S}$ and Meunier, A.: Magnetorheology: fluids, structures and rheology, Ferrofluids, pp. 201-230, springer (2003).

\section{AUTHORS PROFILE}

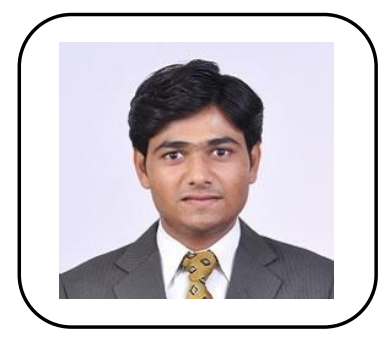

\section{HirenPrajapati}

$\mathrm{He}$ is pursuing his doctoral degree from Nirma University. In the field of smart fluids and structures. He has successfully completed one minor research project funded by Nirma University ((NU/Ph.D/MRP/IT/18 19/538). He has guided 3 M.Techdissertations and more than 20 BTech projects. He has published more than 10 research papers in international conferences and one research paper in national conference.

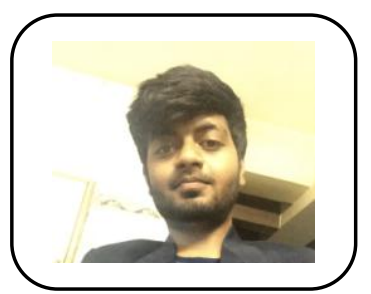

\section{Jas Shahanand}

$\mathrm{He}$ is pursuing his B.Tech degree in Mechanical Engineering Department, Institute of technology Nirma University. $\mathrm{He}$ is doing his B.Tech project in the field of smart fluids since one year under the guidance of Prof. HirenPrajapati.

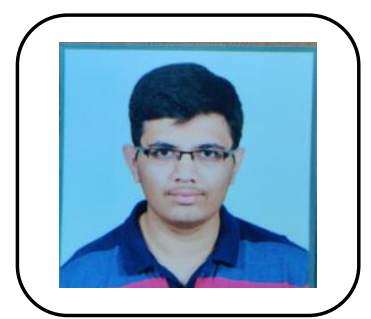

\section{HitarthNimkar}

$\mathrm{He}$ is pursuing his B.Tech degree in Mechanical Engineering Department, Institute of technology Nirma University. $\mathrm{He}$ is doing his B.Tech project in the field of smart fluids since one year under the guidance of Prof. HirenPrajapati. 